\title{
Robustness of Resonance Free Lattices against Gradient Errors
}

\author{
F. Schmidt, A. Verdier (CERN-SL Division), \\ D. Kaltchev (TRIUMF)
}

\begin{abstract}
Resonance-free lattices make it possible to cancel the effect of non-linear resonances due to systematic multipoles in an alternating gradient circular machine. These lattices are made of identical cells with specified phase advances. It is therefore mandatory to examine to what extent the property remains valid in presence of gradient errors. In the case of LHC, three times the nominal gradient errors are acceptable from the point of view of both $\mathrm{a} 3$ and $\mathrm{b} 4$ components.
\end{abstract}

\section{INTRODUCTION}

In AG machines the problem of non-linear resonances excited by systematic multipole components can be solved to a certain extent by using resonance-free lattices [1].

Such a lattice is useful for the LHC at CERN to overcome problems at injection energy associated with unexpected large systematic multipole component in a given fabrication line of the main dipoles. Its efficiency has been demonstrated for the LHC without linear optics errors [2].

The resonance-free condition imposes the phase advances per cell. We report here to which extent gradient errors may destroy this condition. The selected LHC resonance-free lattice has phase advances in the arcs of $\frac{7}{25} \times 2 \pi, \frac{6}{25} \times 2 \pi$ in the horizontal and vertical plane respectively. This is associated with the number of 25 cells in the arcs (23 regular cells plus two special ones for the dispersion suppression). All systematic resonances up to sixth order are cancelled except for the resonance Qy+3 Qx which is fully excited instead.

\section{EVALUATION OF ACCEPTABLE GRADIENT ERRORS}

If the phase advance per cell is changed by gradient errors the resonance terms reappear. However, the benefits of the resonance-free lattice remain valid as long as these resonances are not more important than those due to the random multipole errors. Indeed, in the case of LHC, the random multipoles contribute much less to the dynamic aperture than the systematic multipoles [3]. To compare the resonance driving terms of an arc due to systematic and random components of the same order of magnitude, one has to merely compare the so-called amplification factor [1].

This factor is the ratio of the driving term of an arc to that of a cell. For a pure horizontal resonance of order $n$ it is given by:

$$
\sqrt{\frac{1-\cos \left(N_{c} n \mu_{x, c}\right)}{1-\cos \left(n \mu_{x, c}\right)}},
$$

We set $\mu_{x, c}=\frac{7}{25} \times 2 \pi+\epsilon$, where $\epsilon$ is the difference in phase advance compared to the ideal resonance-free condition. Expanding the amplification factor to first order in $\epsilon$, we obtain $1.9 N_{c} n \epsilon, N_{c}$ being the number of cells per arc.

On the other hand, for a random set of multipole errors the amplification factor is defined as $\sqrt{N_{c} / 6}$, i.e. the incoherent sum of the excitation terms associated with the dipoles in one arc.

These two amplification factors are equal if $1.9 N_{c} n \epsilon=$ $\sqrt{N_{c} / 6}$. For our case, with $n=4$ and $N_{c}=25$, we obtain $\epsilon=0.01=0.0016 \times 2 \pi$. This phase advance corresponds to a systematic b2 of about 6.2 units (unit $=10^{-4}$ at a reference radius of $17 \mathrm{~mm}$ ). The expected value of the systematic b2 is -1.4 units and the uncertainty (systematic per arc) is 0.85 unit. The sum of both absolute values is $2.25 \times 10^{-4}$.

Thus we can probably multiply the present $\mathrm{b} 2$ errors by a factor of value $6.2 / 2.25=2.7$ without destroying the effect of the resonance-free scheme for the b4 component in LHC.

\section{SKEW SEXTUPOLE COMPONENT IN THE MAIN DIPOLES}

The systematic skew sextupole component a3 in the main dipoles can create a large second derivative of the tunes with respect to momentum Q". As explained in Ref. [4], this is a resonant effect. As such it can be cured by the use of a resonance-free lattice.

A large Q" can make a large variation of the chromaticities when the beam energy is changed via a change of the RF frequency, e.g. for a chromaticity measurement. A negative chromaticity Q' may lead to beam loss due to the head-tail instability. In order to prevent the Q' of one unit to become negative for a relative momentum change of 0.001 the value of Q" has to be maintained below 1000 .

We have used normal form analysis ("STATIC" command in the MAD program [5]) to compute the second derivatives of tunes with respect to momentum Q1" and Q2”. We use the standard LHC error table 9901 [6].

The values of the gradient errors from this table have been multiplied by factors between -3 and +3 and applied to the main dipoles and quadrupoles.

For a given multiplication factor we have used 10 realizations of distributions of the systematic a3 errors per arc 
of the main dipoles with a maximum value of 0.9 units. For each realization we applied 10 random distributions of the gradient errors, such that a total of 100 distributions, called seeds in the following, have been treated.

By treating both polarities of the gradient errors the results apply for both rings since the gradients change sign from one ring to the other.
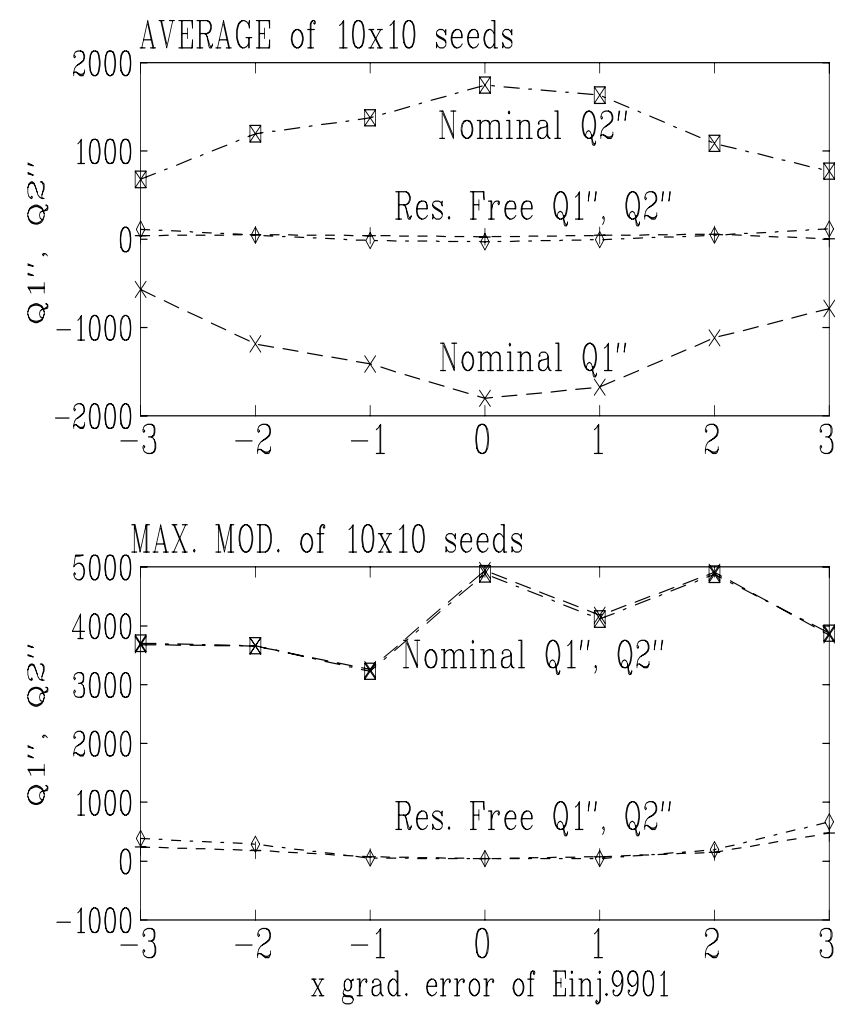

Figure 1: Comparison between the Q" associated with a3 and gradient errors for the nominal and the resonance-free lattices. The 100 seeds of combined errors refer to 10 seeds of systematic a3 and 10 seeds gradient errors. The figures show average (top) and maximum (bottom) of these 100 seeds.

The tune shifts associated with the gradient errors were corrected as in operation by means of the two families of trim quadrupoles which are placed at the end of each arc [7]. It has been checked that a more rigorous correction by means of a rematching of the insertions does not bring any benefits.

The comparison between the nominal and the resonance-free lattice is shown in Fig. 1.
The top plot shows the average and the bottom plot the maximum absolute value of the 100 values of Q1"and Q2". In the case of the resonance-free lattice the maximum allowed value of 1000 for Q" is never exceed for gradient errors up to three times the nominal values.

\section{OCTUPOLE COMPONENT IN THE MAIN DIPOLES}

A large octupole component in the main dipoles may lead to a reduction of the dynamic aperture at injection. In has been shown in Ref. [2] that a substantial reduction of this effect can be obtained by using a resonance-free lattice.

\subsection{Dynamic aperture}

We have performed a tracking study with SixTrack [8] to compare the long term dynamic aperture of the two lattices in presence of increased octupole and gradient errors.

The field errors have been generated using the same error table 9901 and a procedure similar to the one described in the previous section, with two modifications. First, the systematic per arc octupole components have been set to larger than the table values, namely to $b_{4}=1$ and $a_{4}=1.6$ (these are the values used for the study in Ref. [2]). Secondly, the total number of considered cases is reduced to save computing time. The gradient ( $b 2$ ) error of the table is multiplied by factors $-3,-1,0,1$ and 3 (factors \pm 2 omitted) and for each factor and lattice case, 30 (instead of 100) seeds are generated and applied to the main dipoles and quadrupoles.

Table 1 shows the average and minimum dynamic aperture for $10^{5}$ turns. The trajectories start with identical initial conditions in both planes $x_{0}=y_{0}$ and zero values for $x_{0}^{\prime}=y_{0}^{\prime}$. The horizontal and vertical $\beta$-functions at the starting point are $18 \mathrm{~m}$ and $\alpha$-functions are zero.

For a $b 2$-factor equal to zero, the averaged dynamic aperture should be directly compared with the one in Ref. [2]. The slight deviation of $\sim 0.5 \sigma$ is due to the smaller number of error distributions treated.

From the tolerance on the minimum dynamic aperture, we see that a multiplication of the gradient errors by three is acceptable for the resonance free lattice even for such large systematic octupole errors.

\begin{tabular}{||c||c|c||c|c||c|c||c|c||c|c||}
\hline \hline \multicolumn{1}{||c||}{$b_{2} \times$} & \multicolumn{2}{|c|}{-3} & \multicolumn{2}{c|}{-1} & \multicolumn{2}{c|}{0} & \multicolumn{2}{c||}{1} & \multicolumn{3}{c||}{3} \\
\hline & min & ave & min & ave & min & ave & min & ave & min & ave \\
\hline Nominal & 5.7 & 9.6 & 7.8 & 9.9 & 8.1 & 10 & 8.1 & 9.9 & 7.8 & 9.6 \\
\hline Res. free & 9.7 & 12 & 10.8 & 13.3 & 11.4 & 14 & 10.9 & 13.4 & 9.5 & 11.8 \\
\hline
\end{tabular}

Table 1: Minimum and average dynamic aperture (units in $\sigma ; 10^{5}$-turns; $x_{0}=y_{0}$ ) of the nominal and resonance-free lattice for a series of 30 field error distributions. The errors are taken from table 9901 except for the uncertainty (systematic per arc) octupole components which have been set to $b_{4}=1, a_{4}=1$.6. Also the gradient errors have been multiplied by the factor given in the first row. 


\subsection{Resonance driving terms}

Although the dynamic aperture is only weakly correlated with the resonance driving terms, it is still instructive to study their build-up in presence of gradient errors.

The driving terms $h_{i j k l}$ of fourth order nonlinear resonances, generated by skew octupole errors, have been computed to first order with the code SODD [9]. We have concentrated on the skew component since there is a spool piece correction system to compensate the normal octupole component.

\begin{tabular}{||l|c|c|c|c|c|c|c||}
\hline \hline$b_{2} \times$ & -3 & -2 & -1 & 0 & 1 & 2 & 3 \\
\hline Nom. & 12.7 & 7.2 & 2.1 & 5.7 & 5.1 & 4.8 & 4.6 \\
\hline Res.free & 1.1 & 1.0 & 0.7 & 0.4 & 1.0 & 0.6 & 1.2 \\
\hline
\end{tabular}

Table 2: Maximum of the $\mathbf{h} \mathbf{1 0 1 2}$ driving term due to skew octupoles in units of $10^{2}$

We know from previous studies [2] that the most important of the eight diving terms generated by $a 4\left(h_{3010}, h_{3001}\right.$, $\left.h_{2110}, h_{2101}, h_{1030}, h_{1021}, h_{1012}, h_{1003}\right)$, is the term $h_{1012}$.
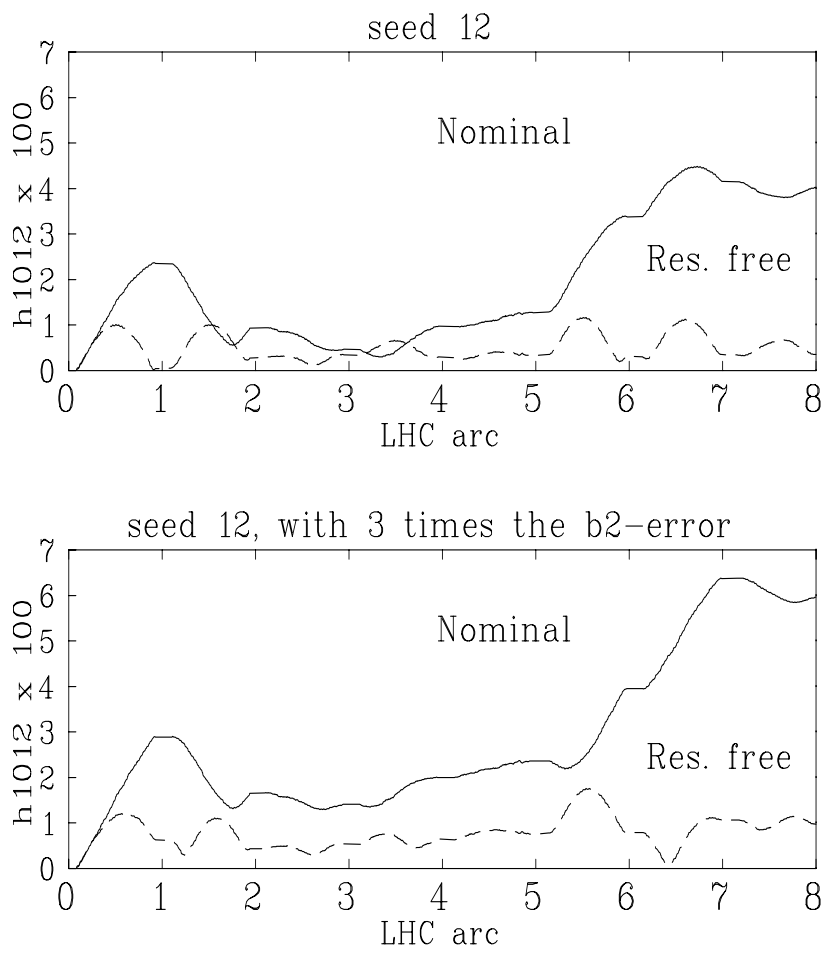

Figure 2: Build up of the driving term $\boldsymbol{h 1 0 1 2}$ along the LHC ring for seed 12

For this term, at zero gradient errors, both the average and maximum values ( 10 seeds) are one order of magnitude smaller for the Resonance free lattice (see Table 2). This remains true even in the presence of gradient error factors.

The build up of this resonance driving term of a particular seed along the LHC ring is depicted in Fig. 2 for both lattices, without (top graph) and with (lower graph) gradient errors. In the resonance-free scheme we find the expected cancellation pattern over each LHC arc while for the nominal lattice the driving term grows on average. This pattern persists in the presence of gradients errors up to three times the nominal values.

As expected, the maximum value of the term $h_{1030}$ is larger for the resonance-free lattice than for the nominal lattice.

\section{CONCLUSION}

We show in this paper that threefold larger gradient errors than nominal do not destroy the benefits of the resonance-free lattice for the LHC with the error table 9901.

For the case of a3, it has been demonstrated that the resonance-free lattice makes it possible to tolerate the nominal a3 error without the a3 correction system even in presence of gradient errors three times as large than nominal.

In the case of b4 and a4 we used 3 and 10 times larger values than their nominal uncertainties and did not take advantage of the b4 correction system. We find that in a window of \pm 3 times the nominal gradient errors the dynamic aperture of the resonance-free lattice is larger than that of the nominal lattice by at least $2 \sigma$, allowing to stay at least above $9.5 \sigma$.

\section{REFERENCES}

[1] A. Verdier, Resonance free lattices for A.G. machines. PAC99, New-York (March 1999). Also CERN-SL-99-018.

[2] F. Schmidt and A. Verdier, Optimisation of the LHC dynamic aperture via the phase advance of the arc cells. PAC99, NewYork (March 1999). Also LHC Project report 297.

[3] M. Böge et al., Overview of the LHC dynamic aperture studies. PAC97, Vancouver (May 1997). Also LHC Project report 106 (June 1997).

[4] S. Fartoukh and J.P. Koutchouk, Chromatic coupling in LHC and its correction. EPAC 2000, 26-30 June 2000, Vienna, Austria.

[5] H. Grote and F.C. Iselin, The MAD program (Methodical Accelerator Design) version 8.16, User's reference manual, CERN/SL/90-13(AP), (rev. 4) (March 27, 1995).

[6] L. Jin, D. Kaltchev, F. Schmidt, A. Verdier, Correction of the systematic b3 error with the resonance-free lattice in the LHC. EPAC2000, Vienna (June 2000). Also LHC Project Report 401.

[7] A. Verdier, Operational Q-shifts and $b 2$ compensation in LHC. LHC Project Note 26 (1996), Unpublished.

[8] F. Schmidt, "SIXTRACK, version 2, single particle tracking code treating transverse motion with synchrotron oscillations in a symplectic manner, CERN SL/94-56 (AP) (1994), http://wwwslap.cern.ch/frs/Documentation/doc.html.

[9] F. Schmidt, "SODD: A Computer Code to calculate Detuning and Distortion Function Terms in First and Second Order", CERN SL/Note 99-009 (AP) (1999). 